\title{
A comprehensive analysis of the epidemiology and clinical characteristics of anti-LRP4 in myasthenia gravis
}

\author{
P. Zisimopoulou ${ }^{\text {a }}$, P. Evangelakou ${ }^{\text {a,b }}{ }^{\text {, J. Tzartos }}{ }^{\text {a }}$, K. Lazaridis ${ }^{\text {a }}$, V. Zouvelou ${ }^{\text {, }}$, \\ R. Mantegazza ${ }^{\mathrm{d}}$, C. Antozzi ${ }^{\mathrm{d}}$, F. Andreetta ${ }^{\mathrm{d}}$, A. Evoli $^{\mathrm{e}}, \mathrm{F}$. Deymeer ${ }^{\mathrm{f}}$, \\ G. Saruhan-Direskeneli ${ }^{\mathrm{f}}$, H. Durmus ${ }^{\mathrm{f}}$, T. Brenner ${ }^{\mathrm{g}}$, A. Vaknin ${ }^{\mathrm{g}}$, S. Berrih-Aknin ${ }^{\mathrm{h}}$, \\ M. Frenkian Cuvelier ${ }^{\mathrm{h}}$, T. Stojkovic ${ }^{\mathrm{h}}$, M. DeBaets ${ }^{\mathrm{i}}$, M. Losen ${ }^{\mathrm{i}}$, P. Martinez-Martinez ${ }^{\mathrm{i}}$, \\ K.A. Kleopa ${ }^{j}$, E. Zamba-Papanicolaou ${ }^{j}$, T. Kyriakides ${ }^{j}$, A. Kostera-Pruszczyk ${ }^{k}$, P. Szczudlik ${ }^{k}$, \\ B. Szyluk ${ }^{\mathrm{k}}$, D. Lavrnic ${ }^{1}$, I. Basta ${ }^{1}$, S. Peric ${ }^{1}$, C. Tallaksen ${ }^{\mathrm{m}}$, A. Maniaol ${ }^{\mathrm{m}}$, S.J. Tzartos ${ }^{\mathrm{a}, \mathrm{b}, *}$
}

${ }^{a}$ Hellenic Pasteur Institute, Athens, Greece

${ }^{\mathrm{b}}$ University of Patras, Patras, Greece

${ }^{c}$ Neurology Department, Aeginition Hospital, Athens, Greece

${ }^{\mathrm{d}}$ Neurological Institute "C. Besta", Milano, Italy

e Institute of Neurology, Catholic University, Rome, Italy

${ }^{\mathrm{f}}$ Istanbul University, Istanbul, Turkey

${ }^{\mathrm{g}}$ Hadassah Hebrew University Medical Center, Jerusalem, Israel

${ }^{\mathrm{h}}$ UPMC and INSERM, Paris, France

${ }^{\mathrm{i}}$ School for Mental Health and Neuroscience, Maastricht University, The Netherlands

${ }^{\mathrm{j}}$ The Cyprus Institute of Neurology and Genetics, Nicosia, Cyprus

${ }^{\mathrm{k}}$ Department of Neurology, Medical University of Warsaw, Poland

${ }^{1}$ Serbia, Clinic of Neurology, Clinical Center of Serbia, Serbia

${ }^{\mathrm{m}}$ Norway Department of Neurology, Ullevaal University Hospital, Oslo, Norway

\section{A R T I C L E I N F O}

\section{Article history:}

Received 29 June 2013

Accepted 8 December 2013

\section{Keywords:}

Myasthenia gravis

LRP4

Autoantibodies

Cell based assay

Diagnosis

Therapy

\begin{abstract}
A B S T R A C T
Double-seronegative myasthenia gravis (dSN-MG, without detectable AChR and MuSK antibodies) presents a serious gap in MG diagnosis and understanding. Recently, autoantibodies against the low-density lipoprotein receptor-related protein 4 (LRP4) have been identified in several dSN-MG sera, but with dramatic frequency variation $(\sim 2-50 \%)$. We have developed a cell based assay (CBA) based on human LRP4 expressing HEK293 cells, for the reliable and efficient detection of LRP4 antibodies. We have screened about $800 \mathrm{MG}$ patient sera from 10 countries for LRP4 antibodies. The overall frequency of LRP4-MG in the dSN-MG group (635 patients) was $18.7 \%$ but with variations among different populations (range 7-32.7\%). Interestingly, we also identified double positive sera: 8/107 anti-AChR positive and 10/ 67 anti-MuSK positive sera also had detectable LRP4 antibodies, predominantly originating from only two of the participating groups. No LRP4 antibodies were identified in sera from 56 healthy controls tested, while 4/110 from patients with other neuroimmune diseases were positive. The clinical data, when available, for the LRP4-MG patients were then studied. At disease onset symptoms were mild ( $81 \%$ had MGFA grade I or II), with some identified thymic changes (32\% hyperplasia, none with thymoma). On the other hand, double positive patients (AChR/LRP4-MG and MuSK/LRP4-MG) had more severe symptoms at onset compared with any single positive MG subgroup. Contrary to MuSK-MG, $27 \%$ of ocular dSN-MG patients were LRP4 antibody positive. Similarly, contrary to MuSK antibodies, which are predominantly of the IgG4 subtype, LRP4 antibodies were predominantly of the IgG1 and IgG2 subtypes. The prevalence was higher in women than in men (female/male ratio 2.5/1), with an average disease onset at ages 33.4 for females and 41.9 for males. Overall, the response of LRP4-MG patients to treatment was similar to published responses of AChR-MG rather than to MuSK-MG patients.
\end{abstract}

(c) 2013 Elsevier Ltd. All rights reserved.

\footnotetext{
* Corresponding author. Hellenic Pasteur Institute, 127 V. Sofias Ave., GR 11521 Athens, Greece. Tel.: +30 2106478844; fax: +30 2106478842

E-mail address: tzartos@pasteur.gr (S.J. Tzartos).
} 


\section{Introduction}

The neuromuscular junction (NMJ), the synapse between the nerve terminal and muscle, is responsible for converting motor nerve signals into muscle contraction. To maximize the efficiency of signal transduction, the acetylcholine receptors ( $A C h R)$, responsible for depolarization in response to nerve stimulation, are clustered at specific sites within the NMJ, forming typical pretzel-like structures [1]. This clustering is mediated by additional muscle membrane proteins, namely the muscle specific kinase (MuSK) and lipoprotein receptor-related protein-4 (LRP4). LRP4 is a member of a family of membrane proteins with central roles in neuronal and synaptic development. It has been shown specifically to be indispensable for normal NMJ development [2].

Myasthenia gravis (MG) is an autoimmune disease affecting the NMJ of skeletal muscles, leading to muscle weakness and fatigability. In the majority of cases ( $\sim 85 \%)$ it is caused by autoantibodies directed against the AChR, thus termed AChR-MG [3]. These autoantibodies hinder normal signalling via complement activation, as they belong mostly to the IgG1 and IgG3 subclasses [4,5], by causing receptor antigenic modulation, or by directly blocking receptor function [6]. In another $\sim 6 \%$ of MG patients autoantibodies are directed against the MuSK protein, MuSK-MG [7]. In this case, however, they are mostly IgG4 subclass, suggesting that a different pathological mechanism compared to AChR-MG is at play [8-10]. The remaining MG patients are described as double-seronegative (dSN-MG), owing to the lack of detectable AChR or MuSK antibodies. However, it has been shown that several of them have low affinity antibodies against AChR [11]. Furthermore, it was recently shown by three different studies that some dSN-MG patients have autoantibodies against LRP4, thus identifying LRP4 as a novel autoantigen in MG [12-14]. However, apparently due to various factors (such as differences in antigen source, the assay used and ethnic origin of the patients) the proportion of LRP4-MG varied dramatically. Clinical data for this novel MGsubgroup have rarely been reported [15].

MG is a heterogeneous disease; for example AChR-MG patients show more thymic pathological findings than MuSK-MG, while they also have differences in the pattern of affected muscles. Furthermore, they can have a varied response to therapy; for instance MuSK-MG patients can demonstrate hypersensitivity when treated with the cholinesterase inhibitor pyridostigmine, while they are highly benefited from plasma exchange (PLEX). It is, therefore, important to characterise the newly identified MG subgroup, and possibly identify unique characteristics, as they could aid in both its diagnosis and management.

In this study we report the screening of about $800 \mathrm{MG}$ sera from 10 different countries for the detection of LRP4 autoantibodies, using a cell based assay (CBA) with human LRP4. We have found the presence of LRP4 antibodies in $\sim 20 \%$ of the screened dSN-MG sera. Furthermore, we provide data with respect to the epidemiology of LRP4-MG, its major clinical features and the effect of treatments that were administered.

\section{Materials and methods}

\subsection{Sample and data collection}

The groups participating in the study contributed mainly sera from dSN-MG patients, mixed with some sera from seropositive MG or non-MG individuals. Serum samples from 809 MG patients (107 AChR-MG, 67 MuSK-MG, 635 dSN-MG), 110 with other neuroimmune diseases (OND) and 90 healthy controls were collected (Table 1). The samples originated from 10 different countries: Norway, the Netherlands, Poland, France, Italy, Serbia, Greece, Cyprus, Turkey and Israel. All sera were obtained with the informed consent
Table 1

Serum samples contributed by each country.

\begin{tabular}{lcccrc}
\hline Country & dSN-MG & AChR-MG & MuSK-MG & OND & Healthy \\
\hline Norway & 43 & 2 & 2 & 0 & 0 \\
The Netherlands & 31 & 0 & 0 & 0 & 0 \\
Poland & 58 & 29 & 5 & 0 & 0 \\
France & 82 & 6 & 3 & 0 & 0 \\
Italy & 66 & 28 & 27 & 30 & 25 \\
Serbia & 45 & 23 & 18 & 0 & 8 \\
Greece & 106 & 19 & 7 & 80 & 57 \\
Cyprus & 64 & 0 & 5 & 0 & 0 \\
Turkey & 69 & 0 & 0 & 0 & 0 \\
Israel & 71 & 0 & 0 & 0 & 0 \\
Total & $\mathbf{6 3 5}$ & $\mathbf{1 0 7}$ & $\mathbf{6 7}$ & $\mathbf{1 1 0}$ & $\mathbf{9 0}$ \\
\hline
\end{tabular}

of the patients. The work has been approved by the Ethical Committee of the Hellenic Pasteur Institute where the anti-LRP4 assays were performed. The anti-LRP4 assays were performed blind. MG was diagnosed based on clinical features and electrophysiological findings. Most Greek dSN-MG patients were selected from the Hellenic Pasteur Institute diagnostic serum biobank on the basis of the short symptom description by their doctors; therefore, these patients are not diagnosed as definitely MG, but as highly likely MG. After the anti-LRP4 assay, the participating teams provided information, when available, for the samples with respect to MG severity, clinical characteristics and therapies, using a template table.

The distribution and severity of myasthenic weakness was classified according to the Myasthenia Gravis Foundation of America (MGFA) grading system. Initial MGFA classification was that of first presentation. Patients' response to therapy was classified according to the MGFA postintervention status (PIS) [16].

\subsection{CBA for the detection of LRP4 autoantibodies}

HEK293 cells transfected with pCMV6-LRP4-tGFP (OriGene) or with pEGFP (Clontech) as a control were fixed $48 \mathrm{~h}$ after transfection with methanol. Fixed cells were incubated with $50 \mathrm{nM} \mathrm{NH} 4 \mathrm{Cl}$ in PBS for $10 \mathrm{~min}$ at room temperature (RT) prior blocking with phosphatebuffered saline containing 5\% BSA (PBS-BSA) and incubated overnight at $4{ }^{\circ} \mathrm{C}$ with patients' sera at 1:100 dilution in PBS-BSA, unless otherwise specified. Alexa Fluor 568-conjugated anti-human IgG antibodies (Life Technologies, Invitrogen) were added at a 1:1000 dilution in PBS-BSA and the samples incubated for $1 \mathrm{~h}$ at room temperature. Rabbit LRP4 antibodies (Santa Cruz Biotechnology, Heidelberg) at a 1:750 dilution and Alexa 568-conjugated antirabbit IgG antibodies (Life Technologies, Invitrogen) at a 1:1000 dilution (both in PBS-BSA) were used for testing the actual LRP4 expression and for the evaluation of each assay. Cells were examined on an Olympus IX51 fluorescence microscope equipped with an Infinity1-2CB digital image system. Initial tests were performed blind, without knowing the autoantibody status and the clinical phenotype of the patients, or which sera were from healthy donors. All sera were evaluated with a score method from 0 : no signal, 0.5 : ambiguous, 1 : weak, 2 : moderate, 3 : strong and 4 : very strong signal by two observers in three independent experiments. The scoring (between observers) did not vary in the case of 0 , whereas there was a variation of $\leq 1$ point in the other sera. Sera with average ambiguous score (0.5) were considered as negative. For the determination of anti-LRP4 IgG subclasses, after incubation with the serum samples, the cells were washed and incubated for $1 \mathrm{~h}$ at room temperature with a 1:50 dilution of sheep anti-human IgG subclass antibodies ( $1 \mathrm{mg} / \mathrm{ml}$; Binding Site, Birmingham), then for $1 \mathrm{~h}$ at room temperature with a 1:1000 dilution of Alexa Fluor 568conjugated donkey anti-sheep IgG antibodies (Life Technologies, 

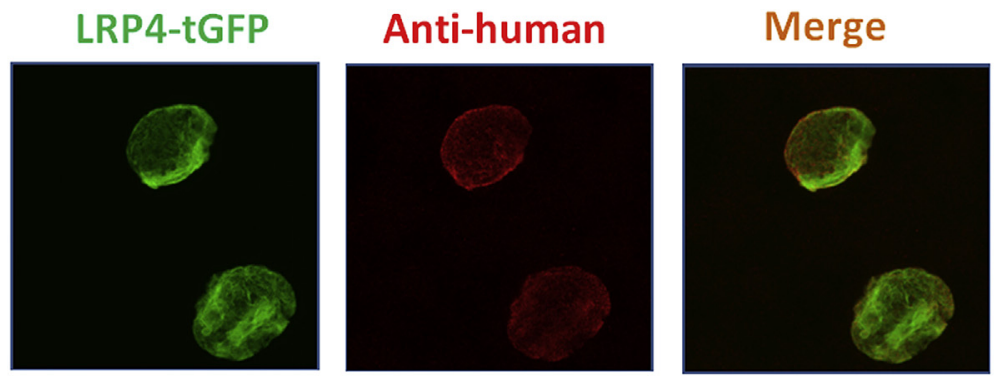

Serum \#1
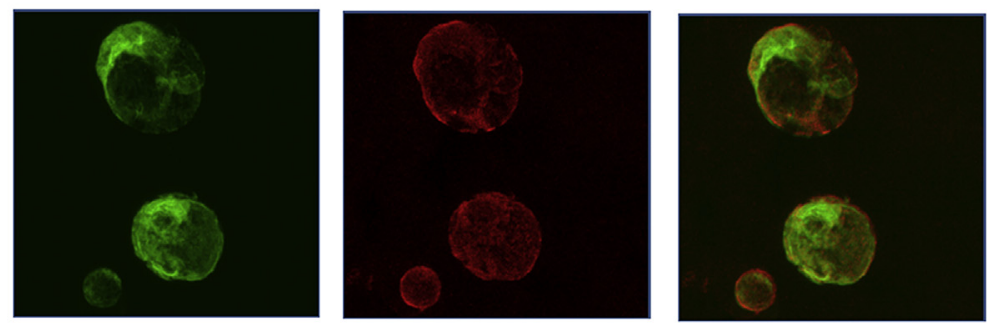

Serum \#2
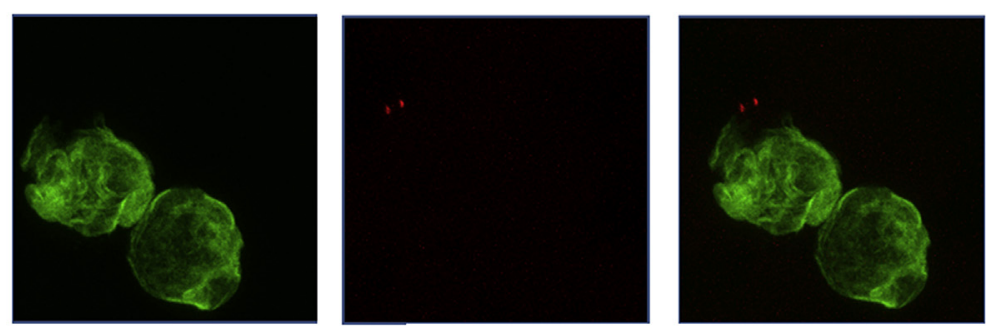

NHS

EGFP
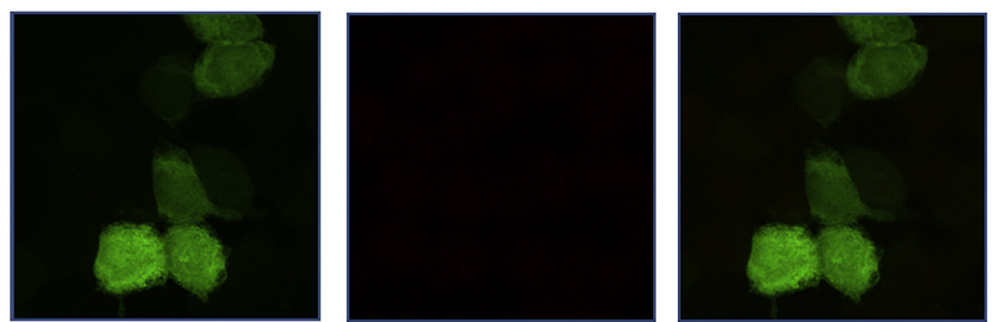

Serum \#1

\section{LRP4-tGFP}

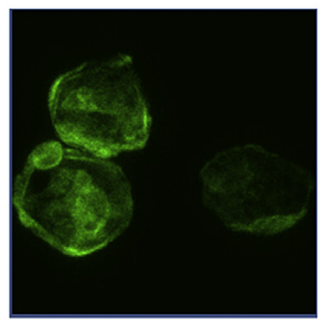

\section{Anti-rabbit}
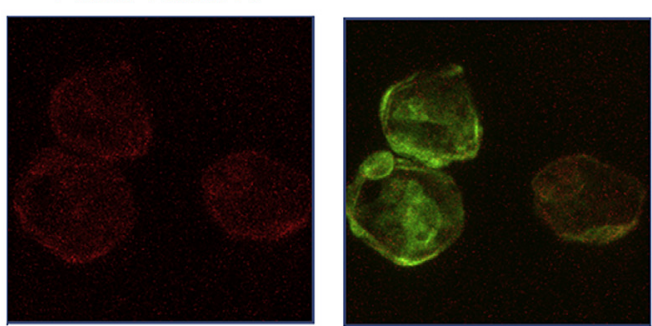

\section{Anti-LRP4}

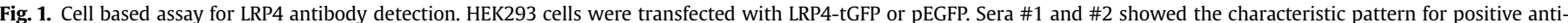

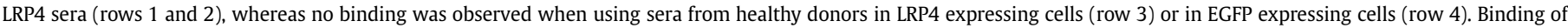

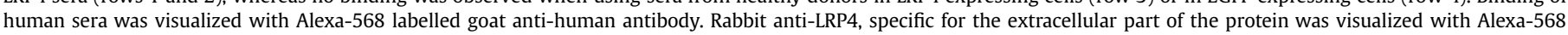
labelled goat anti-rabbit antibody (row 5).

Invitrogen) and examined as described above. Samples were tested $\geq 2$ times, in order to verify the results described under "Results".

\subsection{Radioimmunoprecipitation assays (RIPA) for AChR and MuSK antibodies}

AChR and MuSK antibody titers were determined using the commercial AChR and MuSK antibody RIPA kits (RSR Ltd, Cardiff, UK) according to the manufacturer's instructions. Anti-AChR titers lower than $0.2 \mathrm{nM}$ or higher than $0.5 \mathrm{nM}$ were considered negative and positive, respectively, whereas values between $0.2 \mathrm{nM}$ and $0.5 \mathrm{nM}$ were considered ambiguous. Similarly, anti-MuSK titers lower than $0.02 \mathrm{nM}$ or higher than $0.05 \mathrm{nM}$ were considered negative and positive, respectively, whereas values between 0.02 and $0.05 \mathrm{nM}$ were considered ambiguous. No ambiguous sera were included in this study.

\subsection{Statistical analysis}

Statistical analyses were performed using the student's $t$ test or the Yates' corrected chi-square test. 


\section{Results}

\subsection{LRP4-MG prevalence}

A CBA was developed for the reliable and efficient detection of LRP4 antibodies. In short, HEK293 cells expressing tGFP-tagged intact human LRP4 were incubated with the assayed sera, followed by a second fluorescence labelled anti-human antibody for visualization (Fig. 1). All sera were incubated with the pEGFP transfected cells, which provided a negative control in addition to normal human sera used in each assay. As shown in the merged images of Fig. 1, the co-localization of serum staining with LRP4 was in most cases moderate to high, depending on the serum. The colocalization as evaluated by confocal microscopy for 20 anti-LRP4 sera (including the 2 positive sera of Fig. 1) tested for this reason supported this observation.

To evaluate the specificity of the assay, 90 healthy controls and 110 sera from patients with other neuroimmune diseases (100 of which were multiple sclerosis) were tested. None of the healthy controls was positive, while 4 of the OND samples tested positive (all from multiple sclerosis patients).

Using the developed CBA, we proceeded to screen a number of different MG patient sera from 10 countries (Norway, the Netherlands, Poland, France, Italy, Serbia, Greece, Cyprus, Turkey and Israel). Overall, we found that $18.7 \%$ of the tested dSN-MG sera were positive for LRP4 antibodies (Fig. 2). However, the overall percentage varied among the various populations, from a minimum of $7 \%$ (Norway) to a maximum of $32.7 \%$ (Poland). Interestingly, we also found positives in the AChR-MG and MuSK-MG groups, so that 7.5\% of AChR-MG and 15\% of MuSK-MG patients were double positives for anti-LRP4 (Table 2). Of note, the AChR/LRP4-MG were derived mostly from the Serbian cohort (7/23 Serbian AChR-MG) and only one from the other 84 AChR-MG sera screened. Similarly, half of the MuSK/LRP4-MG came from the Cypriot group (5/5 Cypriot MuSK-MG), whereas the rest came from the other 62 MuSK-MG assayed. There were no known AChR/MuSK-MG double positives in this cohort of patients. From here on LRP4-MG will refer to those patients positive only for LRP4 antibodies, and will not include the double positives. Moreover, sera without detectable autoantibodies against any of the three antigens will be termed triple-seronegative (tSN-MG).

LRP4 autoantibodies appear to favour early onset patients (Fig. 3). Indeed, 65\% of LRP4-MG patients were $<40$ years old at
Table 2

Anti-LRP4 positive sera in AChR-MG, MuSK-MG and controls.

\begin{tabular}{|c|c|c|c|c|}
\hline Sera & & No. & LRP4 + & \% LRP4 + \\
\hline \multirow[t]{7}{*}{ AChR-MG } & Norway & 2 & 0 & 0 \\
\hline & Poland & 29 & 1 & $3.5 \%$ \\
\hline & France & 6 & 0 & 0 \\
\hline & Italy & 28 & 0 & 0 \\
\hline & Serbia & 23 & 7 & $30.5 \%$ \\
\hline & Greece & 19 & 0 & 0 \\
\hline & Total & 107 & 8 & $7.5 \%$ \\
\hline \multirow[t]{8}{*}{ MuSK-MG } & Norway & 2 & 0 & 0 \\
\hline & Poland & 5 & 0 & 0 \\
\hline & France & 3 & 1 & $33.3 \%$ \\
\hline & Italy & 27 & 0 & 0 \\
\hline & Greece & 7 & 1 & $14.3 \%$ \\
\hline & Serbia & 18 & 3 & $16.7 \%$ \\
\hline & Cyprus & 5 & 5 & $100 \%$ \\
\hline & Total & 67 & 10 & $14.9 \%$ \\
\hline OND ${ }^{\mathrm{a}}$ & & 110 & 4 & $3.6 \%$ \\
\hline Healthy & & 90 & 0 & 0 \\
\hline
\end{tabular}

Sera from patients with other neurological diseases.

disease onset, while only $16 \%$ were $>50$ years old (mean onset age 34.9). Early onset was more frequent among the female patients (mean onset age 33.4), whereas males tend to be older at disease onset (mean onset age 41.9) $(P<0.05)$. The female/male ratio within the LRP4-MG group was approximately 2.5:1.

We then characterised the IgG subtype of the LRP4 autoantibodies, using seven randomly selected sera (4 Polish and 3 Greek). Similar to AChR-MG, the majority of antibodies belonged to the IgG1 and IgG2 subtypes, while fewer IgG3 were detected in some of the sera tested (Table 3). The screening was expanded to another sixteen Polish LRP4-MG sera only with respect to the IgG1 and IgG2 isotypes. We found that all sixteen sera had $\operatorname{IgG1}$ and 14/16 had IgG2 type antibodies.

\subsection{Clinical presentation}

We next proceeded to analyse the clinical characteristics of the patients; double positive patients were grouped as separate cohorts. The majority of LRP4-MG patients with available clinical data presented with ocular or mild generalized symptoms, 85\% with MGFA grades I-II at onset, compared to the $15 \%$ with grades III-IV (moderate to severe), while none received a classification of $\mathrm{V}$

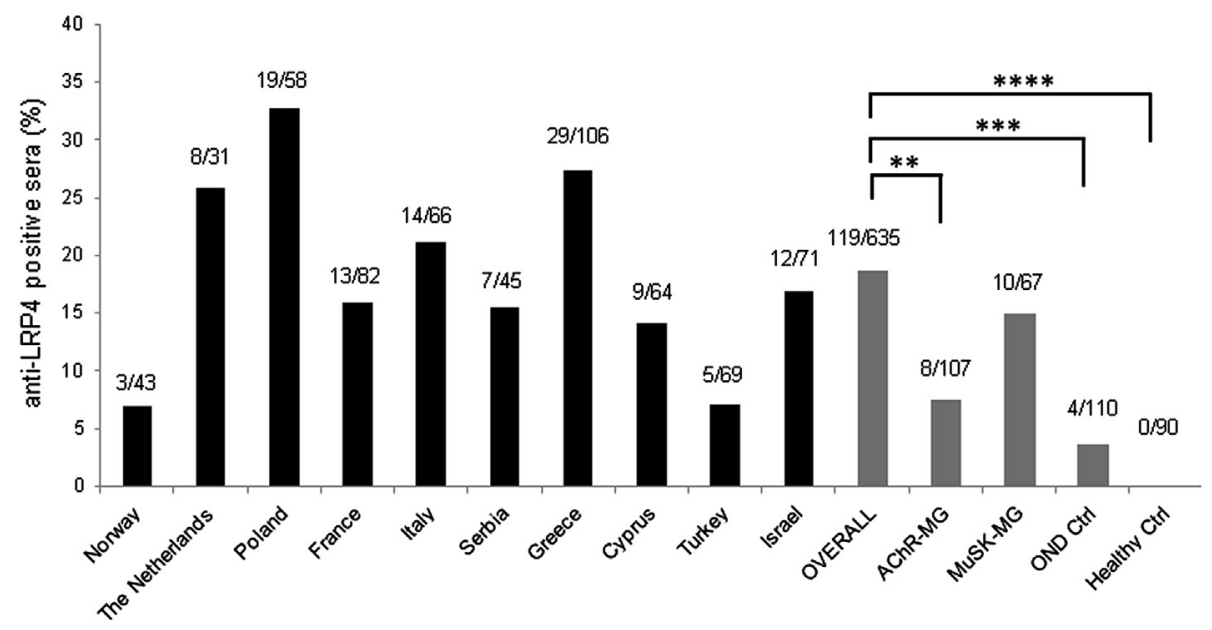

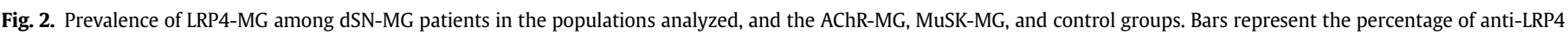
positive patients in the different groups, and numbers above the bars show the number of LRP4-MG patients. $\left({ }^{* *} P<0.01 ;{ }^{* * *} P<0.001 ;{ }^{* * * *} P<0.0001\right)$. 


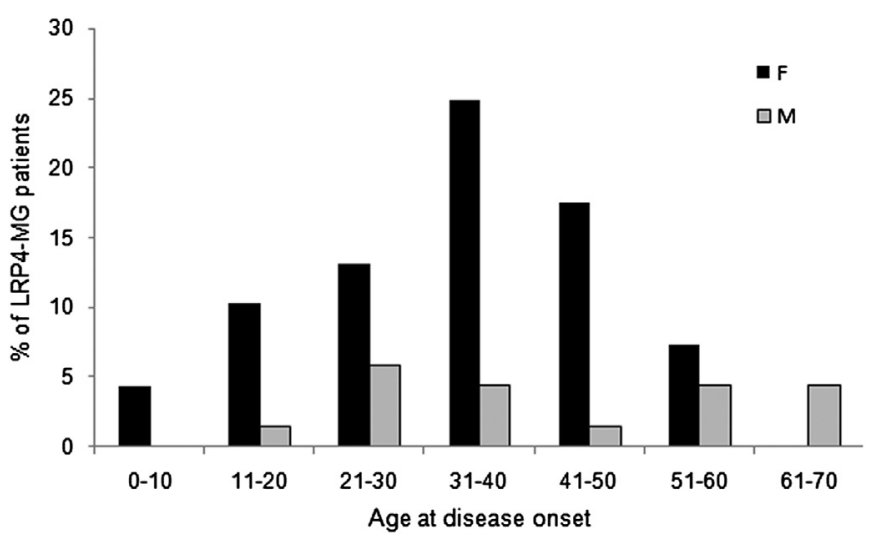

Fig. 3. Age distribution of disease onset among the LRP4-MG patients $(N=68)$.

(Table 4). This is not significantly different from the tSN-MG group, where $81 \%$ were classified MGFA I-II. On the other hand, AChR-MG and MuSK-MG had more severe symptoms at onset $(P<0.01$ and $P<0.05$ respectively compared to the LRP4-MG), with only $51 \%$ and $52 \%$ classified as MGFA I-II at onset. Interestingly, 93\% of the double positive patients (AChR/LRP4-MG or MuSK/LRP4-MG) presented with grade III or IV symptoms. Furthermore, 66\% of LRP4-MG patients presented with bulbar or respiratory muscle involvement, while in $34 \%$ limb or axial muscles were primarily affected. $6.6 \%$ of LRP4-MG and 7.2\% of tSN-MG presented with a myasthenic crisis at some point during their disease. Of note, five of the eight MuSK/ LRP4-MG presented with myasthenic crisis.

Interestingly, patients who remained with only ocular symptoms for more than two years averaged $22.4 \%$ in LRP4-MG and $16.3 \%$ in tSN-MG (Table 4). Moreover, $27 \%$ of the dSN-MG with ocular MG were actually LRP4-MG patients (Table 5). In addition, muscle atrophy has been found in 8\% of LRP4-MG and 3\% of tSN-MG patients (in the majority of cases involving facial and/or pharyngeal muscles).

The thymus is central in AChR-MG pathophysiology and often presents with abnormalities. None of the LRP4-MG patients had developed a thymoma, but thymic hyperplasia (31\%), involuted thymus (29\%) and atrophy (7\%) were reported, while 33\% had normal for age thymi (Table 6). These findings are similar to the tSN-MG group, but LRP4-MG patients appear to have fewer thymic pathologies than the AChR-MG group $(P<0.05)$.

\subsection{Response to therapy}

Among the LRP4-MG patients, a number of different treatments were followed, obviously without previous knowledge of their antiLRP4 seropositivity, with varying responses. Among the most commonly used agents were the acetylcholinesterase inhibitor (AChE-I) pyridostigmine and corticosteroids (prednisone). From the 52 patients treated with pyridostigmine only 5 (9.6\%) did not respond, and similarly, only 4 out of 39 patients (10.2\%) treated with prednisone were not responsive. Therefore, 90\% of LRP4-MG patients showed a positive effect for either one of the treatments,

Table 3

IgG subtype characterization of the LRP4 antibodies.

\begin{tabular}{lllll}
\hline & IgG1 & IgG2 & IgG3 & IgG4 \\
\hline MG sera \#1-7 & $7 / 7$ & $6 / 7$ & $4 / 7$ & $0 / 7$ \\
MG sera \#8-23 & $16 / 16$ & $14 / 16$ & $\mathrm{ND}^{\mathrm{a}}$ & $\mathrm{ND}^{\mathrm{a}}$ \\
Total (as \%) & $100 \%$ & $87 \%$ & $57 \%$ & $0 \%$ \\
\hline
\end{tabular}

${ }^{\mathrm{a}} \mathrm{ND}=$ not determined.
Table 4

Clinical data summary of MG patients. ${ }^{a}$

\begin{tabular}{|c|c|c|c|c|c|c|c|}
\hline MG subgroup & & $\begin{array}{l}\text { LRP4- } \\
\text { MG }\end{array}$ & $\begin{array}{l}\text { AChR- } \\
\text { MG } \\
\left(*^{* *}\right)^{\mathrm{b}}\end{array}$ & $\begin{array}{l}\text { MuSK- } \\
\text { MG } \\
\left({ }^{*}\right)^{\mathrm{b}}\end{array}$ & $\begin{array}{l}\text { AChR/ } \\
\text { LRP4- } \\
\text { MG }\end{array}$ & $\begin{array}{l}\text { MuSK/ } \\
\text { LRP4- } \\
\text { MG }\end{array}$ & $\begin{array}{l}\text { tSN- } \\
\text { MG }\end{array}$ \\
\hline MGFA & I & $20 / 67$ & $7 / 51$ & $3 / 25$ & $0 / 8$ & $0 / 8$ & $74 / 245$ \\
\hline \multirow{4}{*}{ (at first diagnosis) } & II & $37 / 67$ & $19 / 51$ & $10 / 25$ & $1 / 8$ & $0 / 8$ & $124 / 245$ \\
\hline & III & $8 / 67$ & $15 / 51$ & $11 / 25$ & $7 / 8$ & $5 / 8$ & $36 / 245$ \\
\hline & IV & $2 / 67$ & $9 / 51$ & $1 / 25$ & $0 / 8$ & $3 / 8$ & $7 / 245$ \\
\hline & V & $0 / 67$ & $1 / 51$ & $0 / 25$ & $0 / 8$ & $0 / 8$ & $4 / 245$ \\
\hline Ocular $>2$ years & & $15 / 67$ & $4 / 28$ & $1 / 7$ & $0 / 8$ & $0 / 6$ & $40 / 245$ \\
\hline $\begin{array}{l}\text { Bulbar } \\
\text { predominance }\end{array}$ & & $25 / 38$ & $33 / 44$ & $17 / 22$ & $5 / 8$ & $3 / 3$ & $83 / 151$ \\
\hline Myasthenic crises & & $4 / 61$ & $8 / 43$ & $1 / 22$ & $1 / 8$ & $5 / 8$ & $14 / 193$ \\
\hline Muscle atrophy & & $5 / 61$ & $1 / 52$ & $1 / 22$ & $3 / 8$ & $5 / 8$ & 6/193 \\
\hline
\end{tabular}

a Ratios represent the number of patients with a specific characteristic over the total number of patients with available data for that category.

b Comparison of the AChR-MG and MuSK-MG with the LRP4-MG group shows significantly milder symptoms at onset for the LRP4-MG group $\left({ }^{*} P<0.05 ;{ }^{* *} P<0.01\right)$

whereas 5.5\% were not responsive to any of the two (Table 7). Importantly, the tSN-MG patients were also similarly benefited from therapy with pyridostigmine, as only $7 \%$ were not responsive. Although all double positives also benefited by pyridostigmine and prednisone treatments, the MuSK/LRP4-MG appeared more difficult to manage, since approximately half of them responded only moderately to each one of the two therapeutic agents.

From the LRP4-MG patient group, 30\% did not require additional intervention, while $46 \%$ received azathioprine as part of their therapeutic regime. Furthermore, 4/7 AChR/LRP4-MG and 5/7 MUSK/LRP4-MG double positives required the additional administration of azathioprine. A variety of other therapies have been reported, such as mycophenolate mofetil, rituximab, PLEX and intravenous immunoglobulins (IVIg), with the last two being most frequently used, especially in the double positives.

Overall, 35\% of LRP4-MG patients went into remission (complete stable or pharmacological), and a further $44 \%$ showed minimal manifestations or improvement of their clinical status, while there were 3 cases (5.5\%) with reported worsening of their postintervention status (Table 7 ). Moreover, all double positive patients showed signs of improvement or remission. There were no MG-related reported deaths.

\section{Discussion}

We have developed a CBA for the specific detection of LRP4 antibodies, using HEK293 cells expressing the intact human LRP4 protein. The method appears to be specific, since none of the 90 healthy controls that were screened was found positive, while of the 110 samples from patients with other neuroimmune diseases, 4 were found positive. In previous studies, 1 of 101 Lambert-Eaton myasthenic syndrome sera tested, and 2 of 16 neuromyelitis optica patients tested have also been found positive for LRP4

Table 5

Prevalence of LRP4-MG in double seronegative ocular patients.

\begin{tabular}{lcc}
\hline \multirow{2}{*}{ Country } & Ocular MG & \\
\cline { 2 - 3 } & dSN-MG & LRP4-MG \\
\hline Poland & 16 & $5(31 \%)$ \\
Italy & 4 & $4(100 \%)$ \\
Serbia & 15 & $3(20 \%)$ \\
Turkey & 20 & $3(15 \%)$ \\
Total patients & $\mathbf{5 5}$ & $\mathbf{1 5 ( 2 7 \% )}$ \\
\hline
\end{tabular}


Table 6

Thymic pathology.

\begin{tabular}{|c|c|c|c|c|c|c|}
\hline & $\begin{array}{l}\text { LRP4- } \\
\text { MG }\end{array}$ & $\begin{array}{l}\text { AChR- } \\
\text { MG } \\
\left({ }^{*}\right)^{\mathrm{a}}\end{array}$ & $\begin{array}{l}\text { MuSK- } \\
\text { MG }\end{array}$ & $\begin{array}{l}\text { AChR/ } \\
\text { LRP4-MG }\end{array}$ & $\begin{array}{l}\text { MuSK/ } \\
\text { LRP4-MG }\end{array}$ & $\begin{array}{l}\text { tSN- } \\
\text { MG }\end{array}$ \\
\hline $\begin{array}{l}\text { Total } \\
\text { patients }\end{array}$ & 42 & 18 & 19 & 6 & 9 & 152 \\
\hline Normal & $14(33 \%)$ & 0 & $1(5 \%)$ & 0 & $5(56 \%)$ & $76(50 \%)$ \\
\hline Hyperplasia & $13(31 \%)$ & $12(67 \%)$ & $4(21 \%)$ & $4(67 \%)$ & 0 & 33 (22\%) \\
\hline Involuted & $12(29 \%)$ & $5(28 \%)$ & $14(74 \%)$ & $2(33 \%)$ & $4(44 \%)$ & $37(24 \%)$ \\
\hline Atrophy & $3(7 \%)$ & 0 & 0 & 0 & 0 & $2(1.3 \%)$ \\
\hline Thymoma & 0 & $1(5 \%)$ & 0 & 0 & 0 & $4(2.6 \%)$ \\
\hline
\end{tabular}

a Statistical analysis reveals fewer thymic pathologies in the LRP4-MG group compared to the AChR-MG group $\left({ }^{*} P<0.05\right)$.

antibodies [12,13]. The significance of these autoantibodies in other neuroimmune diseases remains to be investigated.

Three teams have shown the presence of LRP4 antibodies in some MG patients [12-14]. However, these studies showed great variability with respect to the occurrence of LRP4 antibodies within the $\mathrm{dSN}-\mathrm{MG}$ population, ranging from 2 to $50 \%$. The very low $2 \%$ of the LRP4-MG in the Japanese population [12] may be due to ethnic variations and/or to the use of solubilised mouse antigen in the assay. Similarly, the moderate $9.2 \%$ anti-LRP4 positivity within the cohort studied by Zhang and co-workers [13] could be influenced by the fact that rat and not human LRP4 was used in the ELISA setup. Finally, the small number of samples examined in the third study prohibits definitive epidemiological analysis of the results [14]. Using the CBA assay described herein, we have performed an international analysis of sera from MG patients, with emphasis on anti-AChR and anti-MuSK seronegative (dSN-MG), aimed at studying the epidemiology of LRP4-MG patients and their clinical presentation. Specifically, we have screened $635 \mathrm{dSN}-\mathrm{GM}$ sera from 10 different countries. Although there was considerable variation among the different populations examined (7-32.7\%), suggesting that environmental and/or genetic factors could contribute to the development of the disease, we could not detect a geographical pattern similar to that of MuSK-MG, with higher prevalence closer to the equator [17]. Recently, Cossins and co-workers [18] using a CBA found 8\% LRP4-MG (among the double seronegatives) and

Table 7

Summary of applied therapies.

\begin{tabular}{|c|c|c|c|c|c|c|}
\hline \multicolumn{3}{|l|}{ MG subgroup } & \multirow{2}{*}{$\begin{array}{l}\text { LRP4- } \\
\text { MG } \\
52 / 60\end{array}$} & \multirow{2}{*}{$\begin{array}{l}\text { AChR/ } \\
\text { LRP4- } \\
\text { MG }\end{array}$} & \multirow{2}{*}{$\begin{array}{l}\text { MuSK/ } \\
\text { LRP4- } \\
\text { MG } \\
8 / 8\end{array}$} & \multirow{2}{*}{$\begin{array}{l}\text { tSN- } \\
\text { MG } \\
154 / 159\end{array}$} \\
\hline Pyridostigmine & Treated/to & & & & & \\
\hline & Response & Good & 40 & 8 & 3 & 128 \\
\hline & & Moderate & 7 & 0 & 5 & 15 \\
\hline & & None & 5 & 0 & 0 & 11 \\
\hline \multirow[t]{4}{*}{ Prednisone } & \multicolumn{2}{|c|}{ Treated/total } & $39 / 60$ & $7 / 8$ & $8 / 8$ & $120 / 159$ \\
\hline & Response & Good & 28 & 6 & 4 & 87 \\
\hline & & Moderate & 7 & 1 & 4 & 11 \\
\hline & & None & 4 & 0 & 0 & 23 \\
\hline Azathioprine & \multicolumn{2}{|c|}{ Treated/total } & $12 / 28$ & $4 / 7$ & $5 / 7$ & $40 / 82$ \\
\hline IVIg & \multicolumn{2}{|c|}{ Treated/total } & $10 / 32$ & $0 / 6$ & $3 / 7$ & $15 / 149$ \\
\hline PLEX & \multicolumn{2}{|c|}{ Treated/total } & $4 / 32$ & $2 / 6$ & $5 / 7$ & $13 / 150$ \\
\hline Thymectomy & \multicolumn{2}{|c|}{ Thymectomised/total } & $27 / 69$ & $5 / 8$ & $0 / 9$ & $67 / 252$ \\
\hline \multirow{6}{*}{$\begin{array}{l}\text { Overall } \\
\text { response } \\
\text { (PIS) }\end{array}$} & \multicolumn{2}{|c|}{ Total } & 55 & 8 & 8 & 115 \\
\hline & \multicolumn{2}{|c|}{ CSR and $\mathrm{PR}^{\mathrm{b}}$} & 19 & 4 & 1 & 34 \\
\hline & \multicolumn{2}{|c|}{$\mathrm{MM}^{\mathrm{c}}$} & 3 & 0 & 0 & 7 \\
\hline & \multicolumn{2}{|c|}{ Improved } & 21 & 4 & 7 & 49 \\
\hline & \multicolumn{2}{|c|}{ Unchanged } & 9 & 0 & 0 & 24 \\
\hline & \multicolumn{2}{|l|}{ Worse } & 3 & 0 & 0 & 3 \\
\hline
\end{tabular}

a Number of patients receiving the specific therapy vs. total patients with available clinical information for each category.

b $\mathrm{CSR}=$ complete stable remission; $\mathrm{PR}=$ pharmacologic remission.

c $\mathrm{MM}=$ minimal manifestations. some double seropositive, mostly MuSK/LRP4-MG. However, no information is available as to the prevalence in individual populations for comparison.

In ocular MG patients, only approximately 50\% have detectable AChR antibodies [19]. In the remaining, MuSK antibodies are rarely detectable [20], and most are dSN-MG. Interestingly, we have shown that $27 \%$ of the studied ocular dSN-MG patients were actually LRP4-MG.

With respect to the other major antigens in MG, we found a higher than anticipated prevalence of double positives, i.e. antiAChR or anti-MuSK positive sera that also had LRP4 antibodies. Curiously, the majority of double positive samples originated from two countries; 7/8 AChR/LRP4 double positives from Serbia and 5/ $10 \mathrm{MuSK} / \mathrm{LRP} 4$ double positives from Cyprus. The samples were randomly selected, so this could be a region-specific finding, requiring further investigation. Nonetheless, there appear to be more frequent double positives with LRP4 antibodies together with AChR or MuSK antibodies, compared to AChR/MuSK double positives which are very rarely reported [21,22].

The age distribution of MG showed distinct patterns. In AChRMG there are two peaks of incidence, an earlier one around the third decade of life with female predominance and a second around the sixth decade with male predominance [23]. In LRP4-MG symptoms present around the fourth decade of life, following a distribution similar to that of MuSK-MG [24]. Furthermore, LRP4MG was found more commonly in women (F/M ratio 2.5/1), albeit less prominently than the 3.6-4/1 female/male ratios reported for MuSK-MG [17,25]. Furthermore, LRP4-MG seems to develop at a younger age in women than men, similar to other MG forms.

At the NMJ, LRP4 acts as a receptor for agrin, causing MuSK dimerisation and activation. LRP4 has been shown to be important for NMJ formation during development [2], but its role in the adult muscle is not clear and consequently, the mode of action of the LRP4 antibodies in MG remains elusive [26]. One possibility is complement activation at the site of the NMJ and destruction of its architecture, similar to the action of AChR antibodies [27]. In agreement with this hypothesis, the majority of the LRP4 antibodies detected in patient sera belonged to the complement fixing IgG1-3 subclasses. Additionally, LRP4 antibodies could induce antigenic modulation of LRP4 molecules, or directly disrupt LRP4 interaction with its signalling partners, as already shown in the case of LRP4-agrin interaction and agrin-induced AChR clustering [12-14]. Further studies are required to fully determine the pathogenicity of these antibodies and how they exert their pathological effect.

The analysis of the clinical characteristics of the LRP4-MG patients revealed its unique features. They have similar MGFA classification distribution at onset as the tSN-MG patients in our cohort, with the majority of patients graded I or II, and only $12 \%$ and $3 \%$ of them with grades III and IV respectively. Moreover, $22.4 \%$ remained ocular, in contrast to the infrequent occasions of ocular MG with MuSK antibodies $[8,17,28]$. Additionally, much fewer LRP4-MG patients presented with myasthenic crises compared to the $25-48 \%$ reported for AChR-MG and MuSK-MG [29,30]. They thus appear to have milder symptoms at onset as well as disease progression, compared to the other single-seropositive patient groups.

Interestingly, all double positive patients presented with severe symptoms from the onset: only 1/16 received an MGFA classification of <III, and 6/16 suffered a myasthenic crisis. Therefore, although for diagnostic purposes detection of antibodies to a single MG antigen would suffice, the finding of LRP4 antibodies together with anti-AChR or anti-MuSK might serve as a marker for increased disease severity.

The thymus has been implicated in the pathophysiology of MG and depending on the MG subgroup thymic pathologies have often 
been reported [31]. AChR-MG and to a lesser extent dSN-MG have been linked to thymic hyperplasia, which, on the other hand, is rare in the case of MuSK-MG [32,33]. Furthermore, thymoma is a scarce finding in MuSK-MG, but up to $15 \%$ of AChR-MG present with coexisting thymoma [3]. We have not found any thymoma cases in LRP4-MG patients, indicating that thymoma might not be common within this MG subtype. Taken together with the low incidence of hyperplasia compared to the AChR-MG group and the absence of pathological findings in one third of the cases studied, it could suggest alternative pathogenesis mechanisms than those at play in AChR-MG.

The management of MG can be difficult, particularly due to the differential responses of the MG subgroups. For example, the effectiveness of thymectomy is especially uncertain in MuSK-MG, due to the rare thymic pathologies seen in these patients, and the possible lack of thymic involvement in its pathogenesis. Furthermore, administration of AChE-Is in MuSK-MG patients not only has limited effect, but can even lead to deterioration of myasthenic weakness. Indeed, $22 \%$ of the recorded MuSK-MG patients in our cohort had an adverse reaction to pyridostigmine (data not shown). LRP4-MG patients responded very well (Table 7), similarly to AChRMG [30], as did the patients from the tSN-MG group. Nonetheless, recurring symptoms were recorded and although 34\% of LRP4-MG achieved remission (complete stable or pharmacological), approximately $70 \%$ of the patients required a combination immunosuppressive therapy for a satisfactory outcome.

In conclusion, by the use of a CBA with human LRP4 we screened a large number of dSN-MG sera from 10 countries, in order to determine the prevalence of LRP4-MG in different populations and identify potential subgroup-specific characteristics in clinical presentation and response to treatment. We found variable frequencies of LRP4-MG in the different countries ranging from $\sim 7$ to $33 \%$. Overall, the clinical presentation was milder than the other MG subgroups, whereas the response to the usual MG therapies was satisfactory. We also found a significant number of double positives (AChR/LRP4-MG and MuSK/LRP4-MG), which in our cohort represented more severe cases than the average singlepositive MG patient. Preliminary identification of the clinical profile of this novel MG subgroup should be useful in the management of MG.

\section{Acknowledgements}

This work was supported by grants from the European Commission (FP7 Fight-MG, Contract No 242210), the Greek NSRF ("Thalis" and "Aristeia"), the Association Francaise contre les Myopathies (AFM), the Muscular Dystrophy Association of the USA (MDA), the Polish-Norwegian Research Fund Grant PNRF 204-AI$1 / 07$ and several national MG associations, including the Cyprus and Greek MG Associations, for their assistance in sera collection.

\section{References}

[1] Marques MJ, Conchello JA, Lichtman JW. From plaque to pretzel: fold formation and acetylcholine receptor loss at the developing neuromuscular junc tion. J Neurosci 2000;20:3663-75.

[2] Weatherbee SD, Anderson KV, Niswander LA. LDL-receptor-related protein 4 is crucial for formation of the neuromuscular junction. Development 2006;133:4993-5000

[3] Meriggioli MN, Sanders DB. Autoimmune myasthenia gravis: emerging clinical and biological heterogeneity. Lancet Neurol 2009;8:475-90.

[4] Lefvert AK, Cuenoud S, Fulpius BW. Binding properties and subclass distribution of anti-acetylcholine receptor antibodies in myasthenia gravis. J Neuroimmunol 1981;1:125-35.
[5] Rodgaard A, Nielsen FC, Djurup R, Somnier F, Gammeltoft S. Acetylcholine receptor antibody in myasthenia gravis: predominance of IgG subclasses 1 and 3. Clin Exp Immunol 1987;67:82-8.

[6] Vincent A, Drachman DB. Myasthenia gravis. Adv Neurol 2002;88:159-88.

[7] Hoch W, McConville J, Helms S, Newsom-Davis J, Melms A, Vincent A. Autoantibodies to the receptor tyrosine kinase MuSK in patients with myasthenia gravis without acetylcholine receptor antibodies. Nat Med 2001;7:365-8.

[8] McConville J, Farrugia ME, Beeson D, Kishore U, Metcalfe R, Newsom-Davis J, et al. Detection and characterization of MuSK antibodies in seronegative myasthenia gravis. Ann Neurol 2004;55:580-4.

[9] Boneva N, Frenkian-Cuvelier M, Bidault J, Brenner T, Berrih-Aknin S. Major pathogenic effects of anti-MuSK antibodies in myasthenia gravis. J Neuroimmunol 2006;177:119-31.

[10] Niks EH, van Leeuwen Y, Leite MI, Dekker FW, Wintzen AR, Wirtz PW, et al. Clinical fluctuations in MuSK myasthenia gravis are related to antigen-specific IgG4 instead of IgG1. J Neuroimmunol 2008;195:151-6.

[11] Leite MI, Jacob S, Viegas S, Cossins J, Clover L, Morgan BP, et al. IgG1 antibodies to acetylcholine receptors in 'seronegative' myasthenia gravis. Brain 2008;131:1940-52.

[12] Higuchi O, Hamuro J, Motomura M, Yamanashi Y. Autoantibodies to lowdensity lipoprotein receptor-related protein 4 in myasthenia gravis. Ann Neurol 2011;69:418-22.

[13] Zhang B, Tzartos JS, Belimezi M, Ragheb S, Bealmear B, Lewis RA, et al. Autoantibodies to lipoprotein-related protein 4 in patients with doubleseronegative myasthenia gravis. Arch Neurol 2012;69:445-51.

[14] Pevzner A, Schoser B, Peters K, Cosma NC, Karakatsani A, Schalke B, et al. AntiLRP4 autoantibodies in AChR- and MuSK-antibody-negative myasthenia gravis. J Neurol 2012;259:427-35.

[15] Zouvelou V, Zisimopoulou P, Rentzos M, Karandreas N, Evangelakou P, Stamboulis E, et al. Double seronegative myasthenia gravis with anti-LRP 4 antibodies. Neuromuscul Disord 2013;23:568-70.

[16] Jaretzki 3rd A, Barohn RJ, Ernstoff RM, Kaminski HJ, Keesey JC, Penn AS, et al. Myasthenia gravis: recommendations for clinical research standards. Task Force of the Medical Scientific Advisory Board of the Myasthenia Gravis Foundation of America. Neurology 2000;55:16-23.

[17] Vincent A, Leite MI, Farrugia ME, Jacob S, Viegas S, Shiraishi H, et al. Myasthenia gravis seronegative for acetylcholine receptor antibodies. Ann N Y Acad Sci 2008;1132:84-92.

[18] Cossins J, Belaya K, Zoltowska K, Koneczny I, Maxwell S, Jacobson L, et al. The search for new antigenic targets in myasthenia gravis. Ann N Y Acad Sci 2012; $1275: 123-8$.

[19] Meriggioli MN, Sanders DB. Muscle autoantibodies in myasthenia gravis: beyond diagnosis? Expert Rev Clin Immunol 2012;8:427-38.

[20] Yang L, Maxwell S, Leite MI, Waters P, Clover L, Fan X, et al. Non-radioactive serological diagnosis of myasthenia gravis and clinical features of patients from Tianjin, China. J Neurol Sci 2011;301:71-6.

[21] Zouvelou V, Kyriazi S, Rentzos M, Belimezi M, Micheli MA, Tzartos SJ, et al. Double-seropositive myasthenia gravis. Muscle Nerve 2013:47:465-6.

[22] Poulas K, Koutsouraki E, Kordas G, Kokla A, Tzartos SJ. Anti-MuSK- and antiAChR-positive myasthenia gravis induced by d-penicillamine. J Neuroimmunol 2012;250:94-8.

[23] Poulas K, Tsibri E, Kokla A, Papanastasiou D, Tsouloufis T, Marinou M, et al. Epidemiology of seropositive myasthenia gravis in Greece. J Neurol Neurosurg Psychiatr 2001;71:352-6.

[24] Guptill JT, Sanders DB, Evoli A. Anti-MuSK antibody myasthenia gravis: clinical findings and response to treatment in two large cohorts. Muscle Nerve 2011;44:36-40.

[25] Evoli A, Tonali PA, Padua L, Monaco ML, Scuderi F, Batocchi AP, et al. Clinical correlates with anti-MuSK antibodies in generalized seronegative myasthenia gravis. Brain 2003;126:2304-11.

[26] Verschuuren JJ, Huijbers MG, Plomp JJ, Niks EH, Molenaar PC, MartinezMartinez P, et al. Pathophysiology of myasthenia gravis with antibodies to the acetylcholine receptor, muscle-specific kinase and low-density lipoprotein receptor-related protein 4. Autoimmun Rev 2013;12:918-23.

[27] Tuzun E, Christadoss P. Complement associated pathogenic mechanisms in myasthenia gravis. Autoimmun Rev 2013;12:904-11.

[28] Evoli A, Bianchi MR, Riso R, Minicuci GM, Batocchi AP, Servidei S, et al. Response to therapy in myasthenia gravis with anti-MuSK antibodies. Ann N Y Acad Sci 2008:1132:76-83.

[29] Oh SJ. Muscle-specific receptor tyrosine kinase antibody positive myasthenia gravis current status. J Clin Neurol 2009;5:53-64.

[30] Evoli A, Padua L. Diagnosis and therapy of myasthenia gravis with antibodies to muscle-specific kinase. Autoimmun Rev 2013;12:931-5.

[31] Marx A, Pfister F, Schalke B, Saruhan-Direskeneli G, Melms A, Strobel P. The different roles of the thymus in the pathogenesis of the various myasthenia gravis subtypes. Autoimmun Rev 2013;12:875-84.

[32] Lauriola L, Ranelletti F, Maggiano N, Guerriero M, Punzi C, Marsili F, et al. Thymus changes in anti-MuSK-positive and -negative myasthenia gravis. Neurology 2005;64:536-8.

[33] Leite MI, Strobel P, Jones M, Micklem K, Moritz R, Gold R, et al. Fewer thymic changes in MuSK antibody-positive than in MuSK antibody-negative MG. Ann Neurol 2005;57:444-8. 